\title{
Chatbot e Análise Conversacional para Recomendação da Aprendizagem Colaborativa na EaD
}

\author{
Antônio J. Moraes Neto ${ }^{1}$, Márcia A. Fernandes ${ }^{2}$, Tel Amiel ${ }^{3}$ \\ ${ }^{1}$ Secretaria de Estado de Educação do DF (SEEDF) - Brasília, DF - Brasil \\ ${ }^{2}$ Universidade Federal de Uberlândia (UFU) - Uberlândia, MG - Brasil \\ ${ }^{3}$ Universidade de Brasília (UnB) - Brasília, DF - Brasil \\ antonio.neto@ifb.edu.br, marcia@ufu.br, amiel@unb.br
}

\begin{abstract}
Conversational agents have been considered for providing support to distance learning. Moreover, since these agents can suggest interactions among students in a Virtual Learning Environment (VLE), they can contribute for the collaborative learning, an important aspect to improve online education. Then, this article presents a systematic literature review that intends to answer questions related to such agents, collaborative learning and Conversational Analysis (CA). The results indicated that the area is promising, and it is possible to point out applications of Artificial Intelligence in Education, such as a CA strategy to identify collaboration in VLE and possibilities for chatbot's intervention in favor of collaborative learning.
\end{abstract}

Resumo. Agentes conversacionais têm sido usados para fornecer suporte à Educação a Distância. Estes podem sugerir interações entre estudantes em Ambiente Virtual de Aprendizagem (AVA), contribuindo à aprendizagem colaborativa, um aspecto importante para melhorar o ensino-aprendizagem on-line. Esta revisão sistemática de literatura pretende responder questões relacionadas a tais agentes, aprendizagem colaborativa e Análise Conversacional (AC). Os resultados indicaram que a área é promissora, apontando para aplicações da Inteligência Artificial na Educação, como uma estratégia de AC para identificar a colaboração em AVA e possibilidades de intervenção do chatbot em prol da aprendizagem colaborativa.

\section{Contextualização}

Aprendizagem colaborativa apoiada por computador, em inglês Computer-Supported Collaborative Learning (CSCL), teve origem nas pesquisas de Computer-Supported Cooperative Work (CSCW), que desde os anos 80 buscou incorporar ações colaborativas e design participativo como parte de sistemas em ambiente de trabalho. Nessa linha, CSCL propõe o desenvolvimento de novas aplicações que propiciem a aprendizagem em grupo e que ofereçam atividades criativas de exploração intelectual e interação social, tendo surgido nos anos 1990 como contraponto à aprendizagem isolada baseada na perspectiva de computador pessoal. $\mathrm{Na}$ aprendizagem colaborativa estudantes não dependem somente da interação direta com conteúdo e docentes, ampliam-se as possibilidades pela conexão aluno-aluno. Assim, aprendem através de suas dúvidas e interesses, ensinando um ao outro, ao mesmo tempo em que podem 
visualizar como os outros estão aprendendo e suas dificuldades, o que demanda um suporte computacional orientado à interação produtiva de forma motivadora, em um contexto que "requer planejamento estratégico, coordenação e a implantação de currículo, pedagogia e tecnologia apropriados" [Stahl et al. 2006 p. 2]. Portanto, um Ambiente Virtual de Aprendizagem (AVA) para oferecer tal suporte deve, além de integrar recursos de comunicação, oferecer apoio à colaboração entre participantes.

Um agente conversacional, também conhecido como chatbot, pode gerar um diálogo com o aprendiz, instigando-o, por exemplo, a acessar certos conteúdos em uma atividade educativa. O chatbot é responsável por realizar uma conversa inteligente, falada ou escrita, com um ou mais humanos, mas há lacunas quanto à sua capacidade de reconhecer o contexto de uma conversa entre pessoas. Então, pode ser promissor incluir aspectos fundamentais da Análise Conversacional (AC) na arquitetura de um chatbot, permitindo sistematizar as características da conversação on-line em ambientes virtuais. Tal forma de conversa é construída pelos interagentes nesses ambientes, por meio de uma tecnologia de suporte à linguagem, estabelecendo "um contexto comum que precisa ser negociado pelos participantes na ferramenta" [Recuero 2012 p. 2].

A AC oferece uma forma de analisar o entendimento produzido por meio da interação, concentrando-se nos métodos pelos quais os interagentes constroem sentido colaborativamente, com o objetivo de produzir um relato de como a compreensão foi alcançada na conversa [Koschmann 2013]. Uma abordagem metodológica de AC pode avaliar tanto a estrutura, conteúdo, papéis e relacionamentos nas trocas de turnos das conversas, quanto as funções discretas dessas trocas, "como interações sociais, entendimento compartilhado e cognição distribuída” [Abraham et al. 2016 p. 77].

A caracterização da conversa on-line proporcionada pela AC pode ser adotada em AVA para definir onde está havendo interação social, indicando possibilidade de colaboração, ou onde não há interação, mas poderia haver a fim de promover a aprendizagem colaborativa. A partir disso, o chatbot então seria capaz de instigar o debate entre estudantes em um AVA e, constatando a interação ou ausência desta, de indicar ao professor onde poderia ocorrer a colaboração. A fim de investigar outras pesquisas cujos resultados contribuam para aclarar tais hipóteses, este artigo foi desenvolvido como uma Revisão Sistemática de Literatura (RSL).

\section{Metodologia da Pesquisa}

Conforme proposto por [Kitchenham 2004], esta RSL está dividida nas etapas planejamento, execução e resultados, tendo como objetivo analisar os retornos das buscas sobre os temas chatbot, AC e aprendizagem colaborativa no contexto da EaD.

\subsection{Planejamento}

A questão de pesquisa (QP) principal foi estabelecida a partir do objetivo desta RSL, mas para apoiar a elaboração da sua resposta foram definidas ainda outras três QPs.

- QP principal: A adoção de agente conversacional com análise conversacional pode realizar a recomendação da aprendizagem colaborativa na EaD?

- QP1: Como agentes conversacionais são utilizados com análise conversacional em ambientes virtuais? 
- QP2: Quais as evidências existentes de que um agente conversacional pode promover a aprendizagem colaborativa em ambientes virtuais?

- QP3: Como um agente conversacional pode atuar como sistema de recomendação, mantendo a autonomia em contexto da $\mathrm{EaD}$ ?

Foram realizadas buscas por artigos científicos, publicados entre 2009 e 2020, com a data limite de 28/04/2020, nas bases ACM Digital Library, Directory of Open Access Journals (DOAJ), IEEE Xplorer Digital Library, Scielo, ScienceDirect e Springer, pois possuem mecanismos de busca online que permitem filtros e contêm artigos revisados por pares relacionados às áreas de conhecimento abordadas: Computação, Educação e Comunicação Social. As buscas foram feitas nos idiomas Espanhol, Francês, Inglês e Português, mas os trabalhos identificados estavam exclusivamente em inglês, para as quais foram usados os termos descritos na Tabela 1.

Tabela 1. Termos usados nas buscas em inglês [autoria própria]

\begin{tabular}{|l|l|}
\hline \multicolumn{1}{|c|}{ Tópico } & \multicolumn{1}{c|}{ Termos de busca } \\
\hline Agente conversacional & ("conversational agent" OR chatbot OR chatterbot) AND \\
\hline Análise conversacional & (conversation* AND analysis) AND \\
\hline Aprendizagem colaborativa & ("collaborative learning" OR "online collaboration") AND \\
\hline Sistema de recomendação & (recommend* AND system) AND \\
\hline Educação a distância & $\begin{array}{l}\text { ("learning management system" OR LMS OR "virtual learning } \\
\text { environment" OR VLE OR e-learning OR "distance education") }\end{array}$ \\
\hline
\end{tabular}

Os critérios para inclusão e exclusão de trabalhos foram definidos considerando as QPs, sendo incluídos aqueles que abordam: (1) o uso de agentes conversacionais com AC em ambientes virtuais; (2) as formas de um chatbot promover a aprendizagem colaborativa em ambientes virtuais; (3) a adoção de um agente conversacional como sistema de recomendação, mantendo a autonomia em contexto da $\mathrm{EaD}$; e (4) a adoção de chatbot, AC, aprendizagem colaborativa e sistema de recomendação. Os trabalhos foram excluídos quando: (1) o texto completo não estiver disponível para acesso pela internet; (2) não for artigo de periódico ou conferência; e (3) for RSL com artigos que foram publicados anteriormente ao período pesquisado.

\subsection{Execução}

A segunda etapa da RSL inclui identificação, seleção e extração dos artigos. A identificação executa o planejamento e obtém a lista de trabalhos. A seleção dos artigos observa a pertinência de cada trabalho, por meio da análise do título, palavras-chave e resumo. A partir dos artigos selecionados são extraídos aqueles a serem lidos integralmente, desde que atendam aos critérios de inclusão e não contemplem os de exclusão. Na Tabela 2 constam os números das bases de pesquisa que permitiram identificação, não havendo retorno em DOAJ, IEEE Xplorer Digital Library e $\underline{\text { Scielo. }}$.

Foram identificados quarenta e sete trabalhos a partir dos termos de busca na Tabela 1. Em três das seis bases pesquisadas foram selecionados quinze artigos por contemplarem ao menos um dos quatro critérios de inclusão. Seis artigos selecionados 
não foram extraídos devido aos critérios de exclusão, dois a partir do primeiro, três considerando o segundo e um relacionado ao terceiro. Foram extraídos nove artigos com os critérios de inclusão, sendo dois para o primeiro [Procter et al. 2018; Uthus and Aha 2013], dois a partir do segundo [Hayashi 2019; Tarouco et al. 2018], três devido ao terceiro [Liu et al. 2019; Pons Lelardeux et al. 2017; Schouten et al. 2017] e dois considerando o quarto [Tawfik et al. 2020; Zawacki-Richter et al. 2019].

Tabela 2. Resultado da etapa de execução [autoria própria]

\begin{tabular}{|l|c|c|c|}
\hline \multicolumn{1}{|c|}{ Fonte } & Identificação & Seleção & Extração \\
\hline ACM Digital Library & 12 & 1 & 0 \\
\hline ScienceDirect & 8 & 2 & 2 \\
\hline Springer & 27 & 12 & 7 \\
\hline Total & $\mathbf{4 7}$ & $\mathbf{1 5}$ & $\mathbf{9}$ \\
\hline Google Acadêmico & 188 & 10 & 0 \\
\hline
\end{tabular}

Visando maior amplitude dos resultados, as buscas foram realizadas no período de onze anos e complementarmente no Google Acadêmico, o qual permitiu a identificação de outros trabalhos além daqueles identificados inicialmente nas bases citadas, resultando na seleção de mais dez artigos, dos quais nenhum foi extraído.

\subsection{Resultados}

Pode-se afirmar que são escassos os trabalhos correlatos à adoção de agente conversacional com AC para a recomendação da Aprendizagem Colaborativa na EaD, fato que indica ser apropriado incluir neste trabalho apontamentos para novas perspectivas de aplicação da IA na Educação (IAED), a partir desta RSL.

\section{Chatbot e AC para Recomendar a Aprendizagem Colaborativa na EaD}

Ferramentas e serviços baseados em IA têm alto potencial para apoiar estudantes, professores e administradores durante o ciclo de vida do aprendiz, propiciando aprendizagem flexível, interativa e personalizada, aliviando professores de encargos para dedicarem-se mais à sua tarefa principal: o ensino humano empático [Zawacki-Richter et al. 2019]. Chatbots podem desempenhar um papel relevante na $\mathrm{EaD}$, sendo uma Tecnologia Educacional (TE) que permite a aceleração e personalização do processo de aprendizagem, além de facilitar e enriquecer o AVA [Liu et al. 2019].

Quanto ao chatbot como ferramenta pedagógica, [Zawacki-Richter et al. 2019] indicaram que aplicativos inteligentes oferecem oportunidades para o design de sistemas e para o apoio à aprendizagem em ambientes adaptáveis e personalizados. Apontam algumas lacunas como a quase ausência de reflexão crítica sobre desafios e riscos, a fraca conexão com perspectivas pedagógicas teóricas e a necessidade de maior exploração de abordagens éticas e educacionais na aplicação da IAED. Desta maneira, enfatizaram que essa área da TE deve também abordar as dimensões pedagógicas, éticas, sociais, culturais e econômicas. Nessa perspectiva e com base nos resultados 
desta RSL, seguem as QPs com as suas respostas, iniciando pelas mais específicas.

\section{QP1: Como agentes conversacionais são utilizados com AC em ambientes virtuais?}

[Uthus and Aha 2013] apontaram que as mensagens de bate-papo possuem características que diferem de outras formas de comunicação de texto, como o uso frequente de abreviações, acrônimos, palavras cortadas, exclusão de pronomes do sujeito da frase, emoticons, abreviação de nome de usuário, remoção de vogais para reduzir o número de pressionamentos de tecla e o uso criativo de letras maiúsculas e minúsculas, ortografia e pontuação para expressar voz, gesto e tom. Observam que a gramática do bate-papo é comparativamente informal e não estruturada, em comparação com o texto tradicional, com os usuários envolvidos em um tom de conversa.

Outra peculiaridade é o bate-papo com múltiplos participantes, que é constituído de segmentos de conversa emaranhados, dificultando a análise das mensagens, se comparado com outros meios semelhantes como microblogs, SMS e mensagens instantâneas. Dessa maneira, [Uthus and Aha 2013] afirmaram que há novos desafios que dificultam a aplicação das técnicas tradicionais de análise das formas típicas da comunicação escrita ao bate-papo com múltiplos participantes, descrevendo então técnicas de IA aplicadas em pesquisas para a AC de chat.

Os autores debatem a importância das pesquisas para $\mathrm{AC}$ de chat, dentre as quais aquelas voltadas para a $\mathrm{EaD}$, destacando que os estudantes mantêm mais o foco no que está sendo discutido, o que exige esforço dos professores para diminuir o tempo destinado à explicação a fim de privilegiar a interação no AVA. Considerando que as técnicas tradicionais são insuficientes para a análise de bate-papo com múltiplos participantes, [Uthus and Aha 2013] citaram investigações em IA que criaram inovações técnicas como: o processamento de recursos da sala de bate-papo a fim de aproveitar informações adicionais sobre estas, sendo usado para encontrar relacionamento entre as mensagens e assim determinar as trocas de turno e detectar o assunto em debate; o desembaraço de segmento, cuja abordagem mais comum ocorre pela aplicação de métodos de agrupamento, ou clustering methods, mas há abordagem baseada em grafos que vinculam mensagens de sucessores e predecessores com arestas indicadoras da força do relacionamento; e a identificação do atributo da mensagem por meio de técnicas de marcação de palavras e mensagens, podendo marcar o ato de diálogo, ou dialogue act tagging, para determinar o tipo de ação em uma frase, por exemplo, saudação, mensagem de sistema ou questionamento.

O desenvolvimento e avaliação de chatbot no contexto da EaD em Psicologia, com abordagem para a intervenção inteligente e o design pedagógico a fim de aprimorar a interação entre estudantes e agentes conversacionais, foram relatados por [Procter et al. 2018] a partir de pesquisa experimental. O Freudbot foi projetado para responder questões pessoais e teóricas em primeira pessoa, simulando a conversa com Sigmund Freud de acordo com regras básicas de conversação, como cumprimentar, tomar a palavra e reparar mal-entendidos. A partir da análise de $\log s$ de conversas anteriores, foi criada a metodologia para detectar comportamentos de interação dos estudantes, classificados em: tryer, caracterizado por aqueles que elaboram frases mais longas com alto valor de abstração; keyworder, característica daqueles que fornecem frases mais curtas com baixo valor de abstração, em que não há repetição de palavras; e morer, 
caracterizando estudantes que repetem expressões do tipo backchannel, as quais são sinais de incentivo ao diálogo como 'ok' e 'entendo'. Um estudante pode exibir mais de um desses comportamentos, o que talvez indique o baixo desempenho do chatbot ao forçar o aprendiz a buscar outra estratégia que retorne as informações desejadas.

Com base na primeira fase, [Procter et al. 2018] então relataram as definições e implementações das estratégias de intervenção do chatbot com o objetivo de manter, aumentar ou restabelecer o envolvimento de estudantes na conversação, dentre elas a identificação: de atitudes de diálogo como backchannels, diante das quais o chatbot deve continuar a narrativa atual; de sentenças mais longas, para as quais o chatbot talvez precise indicar que não foi possível compreender as questões ou comentários e, neste caso, fazer perguntas que busquem clarear o interesse do estudante; e de tentativas repetidas de sentenças sem resposta, revelando possível problema para o estudante obter um conteúdo, sendo necessário que o chatbot assuma o controle da conversa para sugerir tópicos relevantes. Em tempo real, um algoritmo detecta comportamentos conversacionais e outro, ao perceber as detecções, direciona o chatbot a fazer as intervenções adequadas, sempre buscando fornecer ao estudante outras maneiras de interação. As intervenções foram consideradas bem-sucedidas quando incentivaram o engajamento do estudante e o recompensam com informações sobre o domínio.

Agentes conversacionais são utilizados com AC em ambientes virtuais para reconhecerem o contexto da conversação e assim poderem intervir adequadamente. Para tanto, os seguintes aspectos fundamentais da AC são adotados: identificar uma conversa a partir do reconhecimento da troca de turnos entre determinados participantes; reconhecer o segmento de conversa a partir de metadados como o emissor e o momento do envio, se esta estiver embaraçada; e caracterizar a conversa a partir do seu conteúdo, que pode incluir saudação, backchannel, questionamento, mensagem de sistema e alguma reação do participante à interação com o próprio chatbot.

\section{QP2: Quais as evidências existentes de que um agente conversacional pode promover a aprendizagem colaborativa em ambientes virtuais?}

A adoção de chatbots em AVAs, com ênfase nas tecnologias usadas para a implementação destes e nas estratégias pedagógicas para a aprendizagem colaborativa, é analisada por [Tarouco et al. 2018]. Com base na teoria interacionista de Vygotsky, na qual a Zona de Desenvolvimento Proximal (ZDP) é a medida comprobatória de que a aprendizagem é um processo social onde os estudantes influenciam mutuamente o amadurecimento cognitivo, afirmaram que as interações são relevantes para a construção de conhecimento, principalmente as que viabilizam diálogo, cooperação, troca de informações e debate de ideias. De forma mais ampla, os autores indicaram que entidades virtuais, como avatar e chatbot, concebidas para fins pedagógicos são capazes de: fornecer informações acerca de conceitos estudados; apontar para fontes externas de informação; apoiar a solução de divergências; instigar a reflexão mútua; e fornecer apoio emocional, simulando o desempenho de colegas a fim de estimular o potencial do estudante, em acordo com a ZDP. Porém, destacaram que limitações técnicas para o processamento da linguagem natural dificultam o desempenho de chatbots, indicando para mitigar esse problema a automatização da expansão de bases de conhecimento, por meio do tratamento de corpus linguístico para identificar conceitos-chave, e a integração com bases de pesquisa virtuais e bancos de dados externos. 
A adoção de múltiplos chatbots, cada um exercendo função específica no processo pedagógico, foi o foco da pesquisa experimental de [Hayashi 2019], que os denomina Pedagogical Conversational Agents (PCAs). O autor considera os seguintes fatores de aprendizagem colaborativa relevantes para a atuação dos PCAs: eficácia do feedback imediato, apropriado em tarefas de aprendizagem verbal, e do feedback atrasado, vantajoso no aprendizado de conceitos pois permite mais tempo para a metacognição dos estudantes; não causar interrupções durante interações entre estudantes nas atividades de aprendizagem; e que os chatbots podem beneficiar mais as interações entre estudantes do que o desempenho de aprendizagem deles.

O estudo investigou o uso dos PCAs: consultor de comunicação para fornecer conselhos técnicos como "Por que você não faz algumas perguntas?" ou "Por que não fornece alguns backchannels enquanto seu parceiro explica?"; e consultor de explicação que gera respostas sobre como pensar no tópico em questão [Hayashi 2019 p. 249]. A partir de algumas palavras-chave, cada PCA é acionado, sendo classificadas em frases importantes, para o consultor de explicação responder e em problemas, que o consultor de comunicação responde. Os resultados obtidos permitiram concluir que: os estudantes frequentemente fornecem explicações a partir das intervenções dos PCAs; eles puderam aprofundar mais os seus conhecimentos; o uso de PCAs é relevante em projetos de CSCL, sendo essencial aos estudantes que não estão acostumados com esses ambientes e aos que estão iniciando na área de conhecimento em estudo, pois facilitam na produção de linguagem quanto ao emprego do vocabulário técnico.

Um chatbot, além de fornecer informações técnicas e conteúdos educacionais, pode promover a aprendizagem colaborativa em ambientes virtuais por meio de intervenções que contribuam para a construção de conhecimento dos estudantes, seja simulando um colega, seja incentivando o engajamento colaborativo dos colegas.

\section{QP3: Como um agente conversacional pode atuar como sistema de recomendação, mantendo a autonomia em contexto da EaD?}

[Pons Lelardeux et al. 2017] apresentaram, a partir de pesquisa experimental, um sistema de comunicação usado em jogo digital como uma solução apropriada ao ensino do gerenciamento de riscos, tendo como enfoque situações onde estudantes lidam com fatores humanos, precisando aprender a tomar decisões e a tratar erros conjuntamente. O ambiente virtual colaborativo foi desenvolvido para oferecer mecanismos de comunicação e de tomada de decisão a fim de permitir que uma equipe possa construir a representação comum de situações críticas. O sistema de comunicação foi usado para facilitar o jogo, monitorando a conversa para manter os colegas e o professor informados sobre o andamento de suas realizações.

Os autores indicaram que o sistema de comunicação foi bem avaliado por ter permitido a exposição de comportamentos reais em situações críticas, como debate, conflito e irritação, pois foram implementados recursos que viabilizaram a discussão argumentada de todas as questões levantadas, independente se houve convergência ou divergência entre os participantes. "O papel do sistema de comunicação não é fornecer uma maneira utópica automatizada de resolver esses pontos de desacordo, mas explicitá-los para que os estudantes, em equipe, os identifiquem e aprendam a impedir seu surgimento" [Pons Lelardeux et al. 2017 p. 512]. 
O chatbot desenvolvido a partir da arquitetura conceitual proposta por [Liu et al. 2019] foi adotado em contexto de aprendizagem móvel a fim de recuperar informações em um domínio específico, pois perceberam nas referências estudadas lacunas na adoção de chatbots educativos em dispositivos móveis e na análise de influência da usabilidade destes na aprendizagem de estudantes. Dentre os aspectos revelados como importantes para a eficácia dos chatbots, destacaram: uma base de conhecimento vasta acerca do domínio; a experiência positiva por parte dos usuários; e a melhor usabilidade dessa TE, adequada à aprendizagem móvel. Finalmente, os autores destacaram que a TE forneceu informações aos estudantes de forma individualizada, apontando para a relevância de se introduzir um gerenciamento de diálogo que considere comportamentos de conversação no desenvolvimento de chatbots, implementando assim a AC a fim de apoiar outras estratégias educacionais como a aprendizagem colaborativa.

[Schouten et al. 2017] elicitaram requisitos para definição de um AVA adequado ao uso por cidadãos com baixo letramento a fim de apoiá-los na aprendizagem acerca da participação social. É necessário para tanto que o AVA forneça suporte à aprendizagem situada e contextualizada, considerando os seguintes aspectos: cognitivos, fornecendo opções de visualização de conteúdo para facilitar o acesso; afetivos, permitindo aprendizagem individualizada para evitar exposição a fatores como vergonha e medo de julgamento social mediante a baixa autoeficácia; e sociais, promovendo a presença de estudantes e a interação entre eles para facilitar a discussão em grupo, o trabalho em equipe e a formação de conexões sociais significativas. Os autores destacaram que o software de suporte à aprendizagem não é necessariamente menos eficaz a estudantes com baixo letramento do que para estudantes mais letrados, e que os estudantes têm menos probabilidade de evadirem do curso quando se sentem satisfeitos com o AVA.

Um agente conversacional pode atuar como sistema de recomendação, mantendo a autonomia de estudantes em contexto da $\mathrm{EaD}$, quando é implementado como um recurso que viabiliza a discussão entre eles, proporcionando conhecimento sobre o domínio, apoiando a experiência afetiva e social, além de contribuir para a usabilidade adequada do AVA, inclusive em dispositivos móveis.

\section{QP principal: A adoção de agente conversacional com análise conversacional pode realizar a recomendação da aprendizagem colaborativa na EaD?}

A aplicação da IAED foi foco de [Zawacki-Richter et al. 2019], que constataram ser um campo emergente em TE porque carece de apropriação por parte de educadores para que seja amplamente proveitosa ao processo pedagógico, havendo necessidade de mais pesquisas por parte de professores e designers de aprendizagem quanto à adoção da IA no ensino-aprendizagem. Os autores indicaram ainda que, apesar dos avanços relacionados ao poder computacional e à vasta disponibilidade de dados dos estudantes, há pouco avanço das teorias de aprendizagem relacionadas à aplicação da IAED.

[Zawacki-Richter et al. 2019] apontaram o alto potencial que as soluções de IA têm para apoiar estudantes, professores e gestores no ciclo de vida estudantil, mas observaram que é preciso saber como explorar a IAED, tendo em perspectiva os cuidados técnicos e éticos adequados à complexidade da Educação. Os autores destacaram em suas conclusões os desafios e as oportunidades da IAED apontados pela UNESCO quanto às dimensões pedagógica, social e ética, buscando garantir inclusão e 
equidade nesta área por meio de ações como: a formação de professores para a Educação com IA; o desenvolvimento de bons sistemas de informação que propiciem inclusão; e a garantia de ética e transparência quanto aos dados acadêmicos.

Uma plataforma para aprendizagem colaborativa foi analisada, em pesquisa experimental, por [Tawfik et al. 2020], que foi desenvolvida a fim de apoiar: o design de atividades criadas por especialistas a partir da aprendizagem baseada em projetos; a facilitação cognitiva, ou cognitive scaffolding, por parte de professores para resolução de problemas; e a interação entre estudantes. Esse AVA adota a estratégia de fornecer feedback predefinido durante o bate-papo, em que o chatbot, por meio de análise semântica latente, intervém para incentivar o engajamento estudantil na conversa e manter o foco em um aspecto da tarefa. Os professores puderam editar todos os feedbacks para que os comentários do chatbot fossem adequados a cada situação. Os autores consideraram essa abordagem mais flexível e dinâmica, apresentando considerações sobre como os estudantes interagem com o professor e o próprio chatbot.

A adoção de um chatbot com análise conversacional a fim de realizar a recomendação da aprendizagem colaborativa na $\mathrm{EaD}$ inicia pela $\mathrm{AC}$ a fim de identificar a possibilidade de colaboração, o que inclui, além dos aspectos fundamentais indicados na resposta da QP1, o reconhecimento de relacionamento prévio entre estudantes e a existência de debate entre eles, por meio da troca de turnos que evidencie conversa em torno de questionamentos. A partir disso o chatbot pode intervir adequadamente em prol da aprendizagem colaborativa, conforme apontado na resposta da QP2, atuando como sistema de recomendação de acordo com as conclusões da QP3. Na QP principal foi ainda revelada a necessidade de equipe multidisciplinar, considerando a interação da engenharia de software, pedagogia e as demais áreas de conhecimento envolvidas.

\section{Considerações Conclusivas}

Esta RSL traz trabalhos recentes, seis experimentais e três teóricos. Os estudos em que os chatbots fazem intervenção contextualizada atuam, por limitação do processamento de linguagem natural, em conjunto com a AC, apesar de apresentarem variação deste termo e métodos ainda não finalizados. A AC é usada para estudar como a compreensão é construída em ambientes virtuais e torna viável gerar recomendações em prol da aprendizagem colaborativa. Alguns trabalhos apontaram para o uso de tecnologias prontas, mas todos desenvolveram os próprios chatbots para contextos específicos.

Há lacunas que direcionam estudos para: a especificação de arquiteturas abertas de IAED; a integração de IA ao AVA com base em sistemas de recomendação, em consonância com [Aggarwal 2016] ao afirmar que nos sistemas voltados para o conhecimento as recomendações são sugeridas a partir de requisitos do aprendiz, e não no seu histórico de interação; a definição de metodologia para implementar a camada de AC entre AVA e chatbot; a experimentação de abordagens pedagógicas, éticas e sociais na adoção de chatbots em EaD; e a formação de educadores para a aplicação da IAED a fim de impactar positivamente o ensino-aprendizagem.

\section{Referências}

Abraham, J., Kannampallil, T., Brenner, C., et al. (2016). Characterizing the structure and content of nurse handoffs: A Sequential Conversational Analysis approach. 
IX Congresso Brasileiro de Informática na Educação (CBIE 2020)

Anais do XXXI Simpósio Brasileiro de Informática na Educação (SBIE 2020)

Journal of Biomedical Informatics, v. 59, p. 76-88.

Aggarwal, C. (2016). An Introduction to Recommender Systems. In: Aggarwal, C.[Ed.]. Recommender Systems: The Textbook. Cham: Springer I. Publishing. p. 1-28.

Hayashi, Y. (may 2019). Multiple pedagogical conversational agents to support learner-learner collaborative learning: Effects of splitting suggestion types. Cognitive Systems Research, v. 54, p. 246-257.

Kitchenham, B. (2004). Procedures for Performing Systematic Reviews.

Koschmann, T. (2013). Conversation Analysis and Collaborative Learning. In: Hmelo-Silver, C.; Chinn, C.; Chan, C.; O’Donnell, A.[Eds.]. The International Handbook of Collaborative Learning. New York: Routledge Handbooks. p. 149-167.

Liu, Q., Huang, J., Wu, L., Zhu, K. and Ba, S. (6 jul 2019). CBET: design and evaluation of a domain-specific chatbot for mobile learning. Universal Access in the Information Society.

Pons Lelardeux, C., Panzoli, D., Lubrano, V., et al. (apr 2017). Communication system and team situation awareness in a multiplayer real-time learning environment: application to a virtual operating room. The Visual Computer, v. 33, n. 4, p. 489-515.

Procter, M., Lin, F. and Heller, B. (2018). Intelligent intervention by conversational agent through chatlog analysis. Smart Learning Environments, v. 5, n. 1, p. 30.

Recuero, R. (2012). A Conversação como Apropriação na Comunicação Mediada pelo Computador. In: Buitoni, D. S.; Chiachiri, R.[Eds.]. Comunicação, Cultura de Rede e Jornalismo. Edição: 1 ed. São Paulo: Almedina. p. 259-274.

Schouten, D., Smets, N., Driessen, M., et al. (2017). Requirements for a virtual environment to support the social participation education of low-literates. Universal Access in the Information Society, v. 16, n. 3, p. 681-698.

Stahl, G., Koschmann, T. and Suthers, D. D. (2006). Aprendizagem colaborativa com suporte computacional: Uma perspectiva histórica. In: Sawyer, R. K.[Ed.]. Cambridge handbook of the learning sciences. Cambridge, UK: Cambridge University Press. p. 409-426.

Tarouco, L. M. R., Silveira, C. and Krassmann, A. L. (2018). Collaborative Learning with Virtual Entities. In: Zaphiris, P.; Ioannou, A.[Eds.]. Learning and Collaboration Technologies. Design, Development and Technological Innovation. Cham: Springer International Publishing. v. 10924, p. 480-493.

Tawfik, A., Graesser, A. and Love, J. (2020). Supporting Project-Based Learning Through the Virtual Internship Author (VIA). Technology, Knowledge and Learning, v. 25 , n. 2, p. 433-442.

Uthus, D. C. and Aha, D. W. (2013). Multiparticipant chat analysis: A survey. Artificial Intelligence, v. 199-200, p. 106-121.

Zawacki-Richter, O., Marín, V. I., Bond, M. and Gouverneur, F. (dec 2019). Systematic review of research on artificial intelligence applications in higher education - where are the educators? International Journal of Educational Technology in Higher Education, v. 16, n. 1, p. 39. 